\section{Serum 1,3- $\beta D$-Glucan assay in the diagnosis of invasive fungal disease in neonates}

\author{
Cheryl Anne Mackay,1,2 Daynia Elizabeth \\ Ballot, 1,2 Olga Perovic1,3 \\ 1University of the Witwatersrand, \\ Johannesburg; \\ 2Charlotte Maxeke Johannesburg \\ Academic Hospital, Johannesburg; \\ 3Microbiology External Quality \\ Assessment Reference Unit, National \\ Health Laboratory Service, \\ Johannesburg, South Africa
}

\section{Abstract}

Invasive fungal disease is a significant cause of morbidity and mortality in the neonate. The current study aims to assess the 1,3- $\beta$ D-Glucan (BG) assay in a prospective analysis in neonates with suspected fungaemia. A multicentre, prospective cohort study was conducted in Johannesburg, South Africa. The study included 72 neonates with clinically suspected late onset sepsis who were at high risk of fungaemia. A BG assay was performed on each patient and correlated with a sepsis classification based on the full blood count, C-reactive protein and blood culture results as no fungaemia, possible fungaemia, probable fungaemia or definite fungaemia. Sensitivity and specificity of the BG assay at levels of $60 \mathrm{pg} / \mathrm{mL}$ are $73.2 \%$ and $71.0 \%$ respectively and at levels of $80 \mathrm{pg} / \mathrm{mL}$ are $70.7 \%$ and $77.4 \%$ respectively. Positive and negative predictive values at 60 $\mathrm{pg} / \mathrm{mL}$ are $76.9 \%$ and $66.7 \%$ respectively and at $80 \mathrm{pg} / \mathrm{mL}$ are $80.6 \%$ and $66.7 \%$ respectively. The area under the receiver operating curve is 0.753 . The BG assay is a useful adjunct to the diagnosis of invasive fungal disease in neonates. It does, however, need to be considered in the context of the clinical picture and supplementary laboratory investigations.

\section{Introduction}

Neonatal mortality contributes approximately $36 \%$ to under-5 deaths globally. ${ }^{1}$ In South Africa, the neonatal mortality rate is $21 / 1000$ live births with $21 \%$ of these deaths being due to severe infections. ${ }^{2}$ The impact of neonatal sepsis is clearly a significant one. Fungaemia is becoming increasingly important as a cause and is associated with substantial morbidity and mortality in the preterm infant.3,4

Candida species rapidly colonize the skin and mucous membranes of critically ill neonates which can progress to invasive infection. This is particularly so in critically ill neonates who are at increased risk of fungal infection due to immature immune systems, increased permeability of skin and mucous membranes, parenteral nutrition, broad-spectrum antibiotics, central venous catheters, postnatal steroids and mechanical ventilation. 4 This high-risk population could benefit greatly from early diagnosis. The diagnosis of sepsis in neonates, including invasive fungal disease, is difficult as the clinical presentation is often subtle and signs are often nonspecific. 5 There is no single diagnostic test available to reliably confirm or refute sepsis on presentation. Currently diagnosis is based on a combination of clinical features and laboratory investigations such as the full blood count (FBC), C reactive protein (CRP), erythrocyte sedimentation rate (ESR) and procalcitonin (PCT), amongst others.5,6 Definitive diagnosis depends on a positive blood culture in keeping with clinical features and other laboratory investigations. Blood cultures are often negative in neonates even in the presence of sepsis. ${ }^{7}$ This is even more problematic with fungal infections as blood cultures are only positive in approximately $50 \%$ of cases of invasive candidiasis and less than $10 \%$ of invasive aspergillosis. 8,9

$1,3-\beta$-D-Glucan (BG) is a component of the outer wall of a number of fungi including Candida species, Aspergillus species and Pneumocystis jiroveci. The antigen is released into the bloodstream during invasive infection due to such fungi and can be detected by the Fungitell $^{\mathrm{TM}}$ assay. 10 This assay uses enzymes from the Limulus polyphemus amoebocyte lysate and removes bacterial endotoxin-sensitive factor $\mathrm{C}$ from the lysate to form a reagent. BG from patient serum binds to Factor $G$ in the reagent and activates the horseshoe crab coagulation cascade allowing quantitative assay. Values $>80 \mathrm{pg} / \mathrm{mL}$ are considered positive, 60 $80 \mathrm{pg} / \mathrm{mL}$ are considered equivocal (repeat testing recommended) and values less than 60 $\mathrm{pg} / \mathrm{mL}$ are considered negative. 10 The sensitivity and specificity of the BG assay for invasive fungal infections is reported as $69.9 \%$ and $87.1 \%$ respectively. ${ }^{10}$ Studies to date are based on adult haematological, immunocompromised, cancer and surgical patients. Little information is available with regard to neonates. New diagnostic markers are needed to improve the early diagnosis of fungaemia in newborns. The current study comprised a prospective analysis to determine the usefulness of the BG assay for detection of fungal antigen in clinically suspected neonatal invasive fungal disease at three academic Hospitals in Johannesburg.

\section{Objectives}

To determine the 1,3- $\beta$-D-Glucan assay in a prospective diagnostic analysis in neonates
Correspondence: Cheryl Anne Mackay, Department of Paediatrics and Child Health, University of the Witwatersrand, Private Bag X39, Jubilee Road, Parktown, Johannesburg, South Africa. Tel. +27.823930252 - Fax: +27.11.488-4233.

E-mail: cheryl.mackay@wits.ac.za

Key words: neonate, invasive fungal disease, 1,3 $\beta D$-Glucan assay.

Acknowledgements: Dr Khakhu Mathivha and Dr Elizabeth $\mathrm{Ho}$ are gratefully acknowledged for their assistance with data collection at Chris Hani Baragwanath and Rahima Moosa Hospitals respectively. Many thanks to all the neonatal staff at Charlotte Maxeke Johannesburg Academic, Chris Hani Baragwanath and Rahima Moosa Hospitals for assisting with sample collection as well as the laboratory staff at Charlotte Maxeke Johannesburg Academic Hospital for assisting with sample storage and processing.

Contributions: CAM, study design, protocol submission, data collection and interpretation, statistical analysis, writing and revision of manuscript, article submission; DEB, study design, protocol submission, data interpretation, statistical analysis, manuscript critical revision and final approval; OP, study design, protocol submission, data interpretation, manuscript critical revision and final approval.

Funding: this study was funded by a research grant from the Faculty of Health Sciences, University of the Witwatersrand.

Conflict of interest: the authors report no conflicts of interest.

Received for publication: 16 January 2011. Accepted for publication: 17 April 2011.

This work is licensed under a Creative Commons Attribution 3.0 License (by-nc 3.0).

(C) Copyright C.A. Mackay et al., 2011

Licensee PAGEPress, Italy

Pediatric Reports 2011; 3:e14

doi:10.4081/pr.2011.e14

with suspected fungaemia. To correlate possible, probable or definite fungaemia with $B G$ assay results and determine the sensitivity and specificity of the test in neonates.

\section{Materials and Methods}

\section{Study design}

A multicentre, prospective cohort study was conducted including 72 neonates admitted to the neonatal units at Charlotte Maxeke Johannesburg Academic Hospital (CMJAH), Rahima Moosa Mother and Child Hospital and Chris Hani Baragwanath Hospital in Johannesburg. 


\section{Patient selection}

Patients with clinically suspected late onset sepsis, defined as onset of sepsis after 72 hours of life, were identified and classified as no or high risk for invasive fungal disease. Patients were regarded as high risk for invasive fungal disease and were eligible for inclusion in the study if they had 3 or more of the following criteria: i) Low birth weight $(<2500$ g); ii) Duration of hospitalisation > 3 weeks; iii) Prolonged invasive (intermittent positive pressure) ventilation or non-invasive (continuous positive pressure) ventilation (>1 week); iv) Systemic antibiotic exposure $>72$ hours, including poor clinical response to first or second line antibiotic therapy; v) Postoperative patients or patients with abdominal wall defects; vi) Central venous or arterial catheterisation >72 hours; vii) Received total parenteral nutrition (TPN); viii) Splenomegaly; ix) Persistent severe thrombocytopenia (platelet count $<100,000 / \mathrm{mm}^{3}$ despite second line antibiotic treatment).

Patients on systemic antifungal therapy prior to investigation for new onset sepsis were excluded. Patients fulfilling the above criteria were investigated for sepsis as per unit protocol. This included blood investigations (FBC with platelet count, CRP and blood culture) as well as urine cultures, lumbar puncture and radiological investigations where indicated.

\section{Laboratory investigations}

The blood investigations included an FBC with platelet count, CRP and blood culture collected in a FAN blood culture bottle (as per laboratory standard operative procedures). In addition to these investigations, a sample of blood was drawn for a 1,3- $\beta$-D-Glucan assay. The assay was performed using the Fungitell ${ }^{\mathrm{TM}}$ assay kit (Fungitell ${ }^{\circledR}$ Cape Cod) and was performed in the Microbiology Laboratory at CMJAH according to manufacturer instructions. The assay was performed in duplicate on each sample and the average of the results obtained was determined. In the case of extremely discrepant results, the results have been described as uninterpretable. Further investigations such as lumbar puncture and radiological investigations were performed at the discretion of the attending physician. Informed consent was obtained from the primary caregiver before inclusion in the study. This study was approved by the Human Research Ethics Committee of the University of the Witwatersrand (approval number M090211).

\section{Diagnostic criteria for invasive fungal disease}

Based on the above investigations patients were categorised as one of the following: i) no fungaemia: a high index of suspicion for fungaemia including 3 or more risk factors as described above; blood culture negative; white cell count (WCC), platelet count $(<100$ $000 / \mathrm{mm}^{3}$ ) and C-reactive protein (CRP) within normal limits; no evidence of colonisation with a fungal organism; OR definite bacterial sepsis; ii) possible fungaemia: a high index of suspicion for fungaemia including 3 or more risk factors as described above; blood cultures negative; no evidence of colonisation; two or more of the following present: abnormal WCC, low platelet count or elevated CRP (>10); iii) probable fungaemia: a high index of suspicion for fungaemia including 3 or more risk factors as described above PLUS evidence of colonisation with a fungal organism in the form of positive stool or non-sterile urine sample (collected by urine bag) for yeasts; blood cultures negative; and one or more of the following are present: abnormal WCC, low platelet count $\left(<100000 / \mathrm{mm}^{3}\right)$ or elevated CRP (>10); iv) definite fungaemia: positive culture for a fungal organism from a normally sterile site (including blood or tissue culture). The BG results were correlated with the classification as described above, no fungaemia, possible fungaemia, probable fungaemia or definite fungaemia.

\section{Statistical analysis}

The sample comprised 72 neonates. Continuous variables (including birth weight and gestational age) were not normally distributed and have therefore been described as median and range. Categorical variables have been described using proportions. Sensitivity and specificity of the 1,3- $\beta$-D-Glucan assay have been determined and the positive and negative predictive values of the test have been calculated. The categories possible, probable and definite fungaemia were combined and considered diagnostic for fungaemia for the purpose of statistical analysis. The category no infection or confirmed bacterial infection were analysed as no fungaemia. Receiver operating curves have been used to further analyse the results.

\section{Results}

The initial sample included 79 neonates of which 72 formed part of the analysis. Of the seven potential candidates removed from the study, two caregivers refused consent, two samples were rejected at laboratory level (one was contaminated and the other was haemolysed), two samples had a laboratory error and one result was uninterpretable. The sample characterisitics are summarized in Table 1.

Correlation of sepsis category with blood culture results, evidence of colonisation and $1,3-\beta D-G l u c a n$ assay level are presented in Table 2. Fungal culture from a normally sterile site was positive in 10 cases (nine blood samples and one tissue sample). Four of the samples cultured Candida albicans, four cultured Candida parapsilosis, one cultured Sacchyromyces cerevisiae and one blood culture was reported as yeast but not identified. The lowest BG assay in cases with a positive fungal culture was $109 \mathrm{pg} / \mathrm{mL}$ with seven fungal culture positive cases having a BG assay $>500 \mathrm{pg} / \mathrm{mL}$. In the categories possible fun-

Table 1. Sample characteristics $(n=72)$.

\begin{tabular}{|c|c|}
\hline Variable & Number (\%) \\
\hline Male & $45(62.5 \%)$ \\
\hline Female & $27(37.5 \%)$ \\
\hline Birth weight (g) & $1340(720-4600)^{1}$ \\
\hline Gestational age (weeks) & $31(26-40)^{1}$ \\
\hline Surgical intervention² & $34(47.2 \%)$ \\
\hline IPPV3 & $61(84.7 \%)$ \\
\hline CPAP4 & $5(6.9 \%)$ \\
\hline HFOV5 & $3(4.2 \%)$ \\
\hline TPN6 & $49(68.1 \%)$ \\
\hline Central venous / arterial catheter & r $30(41.7 \%)$ \\
\hline Previous antibiotic exposure & $72(100 \%)$ \\
\hline Empiric antifungal therapy & $18(25 \%)$ \\
\hline \multicolumn{2}{|c|}{ 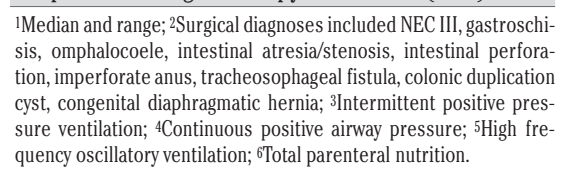 } \\
\hline
\end{tabular}

Table 2. Correlation of sepsis category with blood culture results and $1,3-\beta D-G l u c a n$ assay level $(\mathbf{n}=72)$.

\begin{tabular}{|c|c|c|c|c|c|c|c|}
\hline $\begin{array}{l}\text { Sepsis } \\
\text { categoryl }\end{array}$ & n & $\begin{array}{c}\text { Positive } \\
\text { fungal } \\
\text { culture }^{2}\end{array}$ & $\begin{array}{c}\text { Positive } \\
\text { bacterial } \\
\text { culture }^{2}\end{array}$ & $\begin{array}{l}\text { Evidence of } \\
\text { colonisation }^{3}\end{array}$ & $\begin{array}{l}1,3- \\
<60\end{array}$ & $\begin{array}{l}\text { lucan : } \\
61-80\end{array}$ & $\begin{array}{c}\text { y level } \\
>80\end{array}$ \\
\hline 1 & 31 & 0 & 13 & 7 & 22 & 2 & 7 \\
\hline 2 & 22 & 0 & 0 & 2 & 7 & 1 & 14 \\
\hline 3 & 9 & 0 & 2 & 9 & 4 & 0 & 5 \\
\hline 4 & 10 & 10 & 1 & 3 & 0 & 0 & 10 \\
\hline
\end{tabular}

${ }^{1}$ Sepsis categories as described under methods; ${ }^{2}$ Positive cuture from a normally sterile site (including blood or tissue culture); ${ }^{3 S t o o l ~ o r ~ n o n-~}$ sterile urine sample (urine bag specimen) positive for yeasts. 
gaemia and probable fungaemia, in which cultures from a normally sterile site remained negative and invasive fungal disease would were an additional 19 BG assay levels $>80 \mathrm{pg} / \mathrm{mL}$.

The sensitivity and specificity of the BG assay at levels of $60 \mathrm{pg} / \mathrm{mL}$ are $73.2 \%$ and $71.0 \%$ respectively and at levels of $80 \mathrm{pg} / \mathrm{mL}$ are $70.7 \%$ and $77.4 \%$ respectively. The positive and negative predictive values at $60 \mathrm{pg} / \mathrm{mL}$ are $76.9 \%$ and $66.7 \%$ respectively and at $80 \mathrm{pg} / \mathrm{mL}$ are $80.6 \%$ and $66.7 \%$ respectively. In addition, the area under the ROC curve is 0.753 . Sensitivity, specificity and positive and negative predictive values calculated according to varying BG assay levels are presented in Table 3. R0C statistics and curve are presented in Figure 1.

\section{Discussion}

The current study shows the 1,3- $\beta \mathrm{D}$-Glucan assay to be a useful adjunct in the diagnosis of fungaemia in neonates. Existing recommendations for positivity and negativity of the assay appear to be appropriate for the neonatal period. Despite reasonable sensitivity and specificity of the 1,3- $\beta \mathrm{D}$-Glucan assay, there remain a significant number of false positive and false negative results.

Potential causes of false positive reactivity include patients or specimens exposed to BG containing products (for example gauzes), previously not have been diagnosed, there

patients undergoing haemodialysis with cellulose containing membranes, patients receiving intravenous immunoglobulin therapy and exposure to the antibiotic amoxicillin-clavulanic acid. ${ }^{11}$ Albumin, clotting factors and plasma protein concentrates are manufactured using filters containing high concentrations of BG and may lead to false positivity. In addition, the bacterial organisms Streptococcus pneumoniae and Alcaligenes faecalis are known to contain $\beta$-1,3-glucan in their cell walls and may also cause false reactivity. ${ }^{11}$ Other bacteria, specifically gram positive organisms and Pseudomonas aeruginosa, may also cause false positive reactivity. 12,13

The early diagnosis and treatment of fungaemia, which has been shown to decrease mortality, is challenging. 14 The blood culture as the gold standard of diagnosis is limited by delays of 18 to 72 hours to achieve positivity. ${ }^{8}$ In addition, only $50 \%$ of invasive candidiasis and less than $10 \%$ of invasive aspergillosis are blood culture positive. This curtails the potential survival benefit of earlier antifungal treatment. A reasonable clinical approach to the neonate with suspected fungaemia is to commence antifungal therapy based on a positive 1,3 - $\beta$ D-Glucan assay result (which can be obtained within 24 hours) whilst blood culture results are pending. In the case of a positive

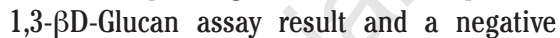
blood culture result one needs to consider both the degree of positivity (for example1,3- $\beta \mathrm{D}$ Glucan assay levels $>250 \mathrm{pg} / \mathrm{mL}$ have a specificity of $96.8 \%$ ) and the presence of factors

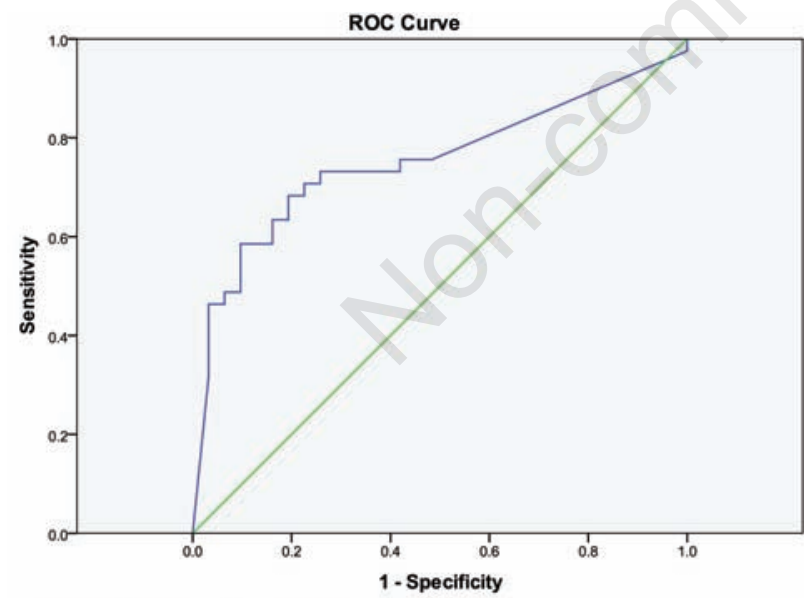

Figure 1. Receiver operating characteristic curve for the 1,3 $\beta D$-Glucan assay.

Diagonal segments are produced by ties. Area under the ROC curve $=0.753$

Table 3. Sensitivity, specificity and positive and negative predictive values at varying $1,3-$ $\beta D-G l u c a n$ assay levels $(n=72)$

\begin{tabular}{ccccc}
$\begin{array}{c}\text { Positive if 1,3- } \beta \mathrm{D}-\text { Glucan } \\
\text { greater than or equal to }\end{array}$ & Sensitivity & Specificity & PPV1 & NPV2 \\
60 & 0.732 & 0.710 & 0.769 & 0.667 \\
80 & 0.707 & 0.774 & 0.806 & 0.667 \\
\hline 150 & 0.585 & 0.839 & 0.828 & 0.605 \\
200 & 0.512 & 0.903 & 0.875 & 0.583 \\
\hline 500 & 0.317 & 0.968 & 0.923 & 0.517 \\
\hline
\end{tabular}

causing false positive reactivity. Results also need to be interpreted taking the infant's clinical presentation into consideration.

One of the difficulties in the current study was the criteria used for diagnosing fungaemia. Use of the blood culture as gold standard would be ideal but the yield of positive blood cultures in neonatal sepsis is known to be low. 7 This led to the use of alternative criteria for the diagnosis of fungaemia which, although unavoidable, may lead to inaccuracies in the analysis. A similar dilemma surrounding diagnostic criteria is demonstrated in a previous study by Odabasi et al. ${ }^{15}$ In this study sensitivity and specificity of the BG assay at a level of $60 \mathrm{pg} / \mathrm{mL}$ are reported as $100 \%$ and $90 \%$ respectively for proven or probable fungaemia and $70 \%$ and $96 \%$ respectively for proven, probable or possible fungaemia.

Limitations of the BG assay include the cost of the test (particularly important in a developing country such as South Africa) and the lack of species identification. A positive BG assay result indicates the presence of one of a number of possible fungi, including amongst others Candida species, the most common fungal isolate in neonates. ${ }^{3}$ It is, however, dependent upon the blood culture for identification and sensitivity of the causative organism. For this reason the BG assay may be of particular use in an epidemic or nosocomial outbreak setting.

\section{Conclusions}

The BG assay is a useful adjunct to the diagnosis of fungaemia in neonates with a predictive value of 0.753 on ROC analysis. BG varies in specificity, depending on the cut-off values used, due to a number of false positive results. The assay therefore needs to be considered in the context of the clinical picture and supplementary laboratory investigations. Ongoing research is required into further causes of false positive reactivity and the change in assay levels in response to treatment.

\section{References}

1. UNICEF. The State of the World's Children 2008: Child Survival. 2008; Available from: http://www.unicef.org/publications/files/Th e_State_of_the_Worlds_Children_2008.p df. Accessed 06/10, 2009.

2. WHO. World Health Statistics 2006. 2006; Available from: http://www.who.int/whosis/en/. Accessed 31 January, 2009.

3. Couto RC, Carvalho EA, Pedrosa TM, et al. A 10-year prospective surveillance of nosocomial infections in neonatal intensive care units. Am J Infect Control 2007;35: 
183-9.

4. Kaufman D, Boyle R, Hazen KC, et al. Fluconazole prophylaxis against fungal colonization and infection in preterm infants. N Engl J Med 2001;345:1660-6.

5. Arnon S, Litmanovitz I. Diagnostic tests in neonatal sepsis. Curr Opin Infect Dis 2008; 21:223-7.

6. Ballot DE, Perovic 0, Galpin J, Cooper PA. Serum procalcitonin as an early marker of neonatal sepsis. S Afr Med J 2004;94:851-4.

7. Kaufman D, Fairchild KD. Clinical microbiology of bacterial and fungal sepsis in very-low-birth-weight infants. Clin Microbiol Rev 2004;17:638-80.

8. Ostrosky-Zeichner L, Alexander BD, Kett $\mathrm{DH}$, et al. Multicenter clinical evaluation of the (1-->3) beta-D-glucan assay as an aid to diagnosis of fungal infections in humans. Clin Infect Dis 2005;41:654-9.

9. Einsele H, Hebart H, Roller G, et al. Detection and identification of fungal pathogens in blood by using molecular probes. J Clin Microbiol 1997;35:1353-60.

10. Kedzierska A, Kochan P, Pietrzyk A, Kedzierska J. Current status of fungal cell wall components in the immunodiagnostics of invasive fungal infections in humans: galactomannan, mannan and (1->3)-beta-D-glucan antigens. Eur J Clin Microbiol Infect Dis 2007;26:755-66.

11. Mennink-Kersten MA, Verweij PE. Nonculture-based diagnostics for opportunistic fungi. Infect Dis Clin North Am 2006; 20:711-27.

12. Pickering JW, Sant HW, Bowles CA, et al. Evaluation of a (1->3)-beta-D-glucan assay for diagnosis of invasive fungal infections. J Clin Microbiol 2005;43:595762 .

13. Mennink-Kersten MA, Ruegebrink D, Verweij PE. Pseudomonas aeruginosa as a cause of 1,3-beta-D-glucan assay reactivity. Clin Infect Dis 2008;46:1930-1.

14. Morrell M, Fraser VJ, Kollef MH. Delaying the empiric treatment of candida bloodstream infection until positive blood culture results are obtained: a potential risk factor for hospital mortality. Antimicrob Agents Chemother 2005;49:3640-5.

15. Odabasi Z, Mattiuzzi G, Estey E, et al. BetaD-glucan as a diagnostic adjunct for invasive fungal infections: validation, cutoff development, and performance in patients with acute myelogenous leukemia and myelodysplastic syndrome. Clin Infect Dis 2004;39:199-205. 